\title{
BDNF Levels and Genotype are Associated with Antipsychotic-Induced Weight Gain in Patients with Chronic Schizophrenia
}

\author{
Xiang Yang Zhang, ${ }^{* 1,2,3}$, Dong Feng Zhou ${ }^{*, 2}$, Gui Ying Wu ${ }^{1,3}$, Lian Yuan Cao ${ }^{3}$, Yun Long Tan ${ }^{3}$, Colin N Haile', \\ Jun $\mathrm{Li}^{4}$, Lin Lu ${ }^{5}$, Therese A Kosten' and Thomas R Kosten ${ }^{\mathbf{1}}$ \\ 'Menninger Department of Psychiatry and Behavioral Sciences, Baylor College of Medicine, Houston, TX, USA; ${ }^{2}$ Institute of Mental Health, \\ Peking University, Beijing, PR China; ${ }^{3}$ Center for Biological Psychiatry, Beijing HuiLongGuan Hospital, Beijing, PR China; ${ }^{4}$ College of Pharmacology, \\ Anhui Medical University, Hefei, Anhui Province, PR China; ${ }^{5}$ National Institute on Drug Dependence, Peking University, Beijing, PR China
}

Recent evidence suggests that centrally released brain-derived neurotrophic factor (BDNF) modulates eating behavior and metabolism that is responsible for body weight fluctuation. BDNF also may play an important role in the therapeutic action of antipsychotic medications. We investigated whether the Val66Met polymorphism of the BDNF gene affected weight gain after long-term antipsychotic treatment in schizophrenia. The polymorphism was genotyped in 196 Chinese patients with schizophrenia on long-term antipsychotic medication. Serum BDNF was measured in all patients and 50 normal controls. Mean body mass index (BMI) change was evaluated retrospectively by means of clinical records. The results showed that there was a significant relationship between the three BDNF Val/ Met genotypes and mean BMI gain, with genotype having a strong effect on BMI gain in male but not female patients. BDNF levels were significantly lower in patients than normal controls, and negatively correlated with BMI gain in female but not male patients. Our results suggest that variation in the BDNF gene may be a risk factor for weight gain in male patients with schizophrenia on long-term antipsychotic treatment, and decreased BDNF levels may be associated with weight gain in females.

Neuropsychopharmacology (2008) 33, 2200-2205; doi:10.1038/s..npp.1301619; published online 7 November 2007

Keywords: schizophrenia; antipsychotic; weight gain; BDNF; genotype; association

\section{INTRODUCTION}

Antipsychotic pharmacotherapy is associated with considerable weight gain and varies by the specific medication, with clozapine and olanzapine having the greatest potential for weight gain (Allison et al, 1999; Allison and Casey, 2001; Baptista, 1999; Correll and Malhotra, 2004; Nasrallah, 2003; Taylor and McAskill, 2000). Antipsychotic-induced weight gain is a leading cause of discontinuation of pharmacotherapy and risk for relapse (Werneke et al, 2002; Nasrallah, 2003). Weight gain is also linked to greater morbidity and mortality as well as psychological well-being (Blackburn, 2000; Nasrallah, 2003).

*Correspondence: Dr XY Zhang, Menninger Department of Psychiatry and Behavioral Sciences, Baylor College of Medicine, VA Medical Center, Research Building 109, Room 130, 2002 Holcombe Boulevard, Houston, Texas 77030, USA, Tel: + | 7|3-79|-|4|4 ext. 5824, Fax: + | 7|3-794-7938, E-mail: xyzhang@bcm.edu

and Dr DF Zhou, Institute of Mental Health, Peking University, 5 I HuaYuan-Bei Road, Hai-Dian District, Beijing I00083, PR China, Tel: + 86 10 82801998, Fax: +86 10 62027314, E-mail: zhoudf@bjmu.edu.cn Received 23 May 2007; revised 3 August 2007; accepted 3 October 2007
Brain-derived neurotrophic factor (BDNF) and its tyrosine kinase receptor, TrkB, are strongly implicated in weight regulation and expressed in hypothalamic nuclei that are associated with eating behavior, food consumption, and control of body weight (Kernie et al, 2000). For example, $\mathrm{BDNF}$ heterozygous mice and mice in which the BDNF gene has been deleted in the brain's excitatory neurons are obese (Kernie et al, 2000; Lyons et al, 1999; Rios et al, 2001). Both central and peripheral administration of BDNF decreases food intake, increases energy expenditure, ameliorates hyperinsulinemia, and hyperglycemia, and reduces weight in diabetic $d b / d b$ mice (Nakagawa et al, 2000; Nonomura et al, 2001; Ono et al, 1997; Tsuchida et al, 2001). Interestingly, a recent study demonstrated a strong association of the Val66Met BDNF variant with a number of eating disorders (Ribases et al, 2003, 2004). Taken together, these findings suggest that BDNF may regulate eating behavior, energy homeostasis, and body weight. Furthermore, both atypical (such as clozapine and risperidone) and typical antipsychotic medications decrease BDNF concentrations in the hippocampus and both frontal and occipital cortices (Angelucci et al, 2000; Lipska et al, 2001). Thus, lowered BDNF levels should be expected in schizophrenics, particularly those with neuroleptic-induced 
weight gain. We therefore compared serum levels of BDNF in patients with chronic schizophrenia to control subjects.

In addition, we examined a potential functional single nucleotide polymorphism (SNP) in the promoter of the BDNF gene at codon 66 (val66met) that might make some schizophrenics more susceptible to weight gain from neuroleptics. This BDNF polymorphism dramatically alters the intracellular trafficking and packaging of pro-BDNF and, thus, the regulated secretion of the mature peptide (Egan et al, 2003). However, to our knowledge, no studies have evaluated the effect of this val66met polymorphism on BDNF serum levels in humans. Therefore, we selected a representative sample of Chinese in-patients with schizophrenia undergoing supervised long-term antipsychotic treatment to ensure sustained medication adherence in examining these metabolic effects. We wanted to determine (1) whether antipsychotic-induced weight gain was associated with BDNF genotype and (2) whether this genotype was related to serum BDNF levels.

\section{MATERIALS AND METHODS}

\section{Subjects}

All subjects were in-patients of Beijing Hui-Long-Guan Psychiatric hospital who had been treated with antipsychotics for at least 10 years and had a normal baseline bodyweight with a body mass index (BMI) of $18-23 \mathrm{~kg} / \mathrm{m}^{2}$ before initiating antipsychotic treatment and met DSM-IV criteria for schizophrenia (American Psychiatric Association, 1994) by agreement of two senior psychiatrists, using the Structured Clinical Interview for DSM-IV. Initial body weight and BMI (weight in kilograms)/(height in meters ${ }^{2}$ ) were recorded from the clinical records, when these firstepisode schizophrenics were drug-naive. Data from the clinical records also included age, gender, education, age of onset, age at antipsychotic initiation, duration of illness, period of antipsychotic treatment, lifetime antipsychotic dose, and family history of psychosis.

From the initial sample of 208 patients, five were excluded due to diabetes mellitus and two due to endocrinopathies. In addition, we excluded five patients with a baseline BMI of less than 18 or more than 23 before initiating antipsychotic treatment. The patients had a mean duration of illness of $22 \pm 7$ years, a mean duration of hospitalization of $10 \pm 7$ years, and an average duration of antipsychotic treatment of $18 \pm 6$ years with a minimum of 2 years treatment on their current antipsychotic. Antipsychotic treatment consisted mainly of monotherapy with clozapine $(n=98)$, risperidone $(n=36)$, perphenazine $(n=20)$, haloperidol $(n=19)$, chlorpromazine $(n=14)$, fluphenazine, and trifluperazine $(n=9)$. Mean antipsychotic dose (as chlorpromazine equivalents) was $425 \pm 342 \mathrm{mg} /$ day. The average duration of the current antipsychotic treatment was $6 \pm 2$ years at the time of the investigation. In addition, 46 patients received one $(n=39)$ or two $(n=7)$ anti-parkinsonian drugs. In addition to the antipsychotics, 12 patients were taking other psychotropic medications: 8 on lithium and 4 on valproic acid.

Every subject had been in a relatively controlled inpatient environment since initiation of the current antipsychotic treatment, and body weights had been assessed regularly over the 10 or more years of confinement. The controlled environment from the time of admission included dietetically balanced hospital meals (daily energy intake for men, $2500 \mathrm{kcal}$; for women, $2200 \mathrm{kcal}$ ), which were occasionally supplemented by gifts (usually fruit). Patients had the opportunity of physical exercise for an hour every day.

A complete medical history and physical examination, laboratory tests including a urine and blood screen, and electrocardiogram were obtained from all participants. All patients were without acute or chronic illnesses known to affect the immune, endocrine, or metabolic systems including diabetes or eating disorders, and none suffered from alcohol or illegal drug abuse/dependence. After a complete description of the study, all subjects gave their written informed consent to participate in the study. The protocol was approved by the Institutional Review Board of the Institute of Mental Health, Peking University.

Fifty healthy controls (34 males and 16 females) without current or past psychiatric history were selected from the local community and gave similar informed consent to be studied. Psychiatric disorders were ruled out among controls by a psychiatric interview conducted by a psychiatrist. All subjects were Han Chinese recruited from the Beijing area. Both patients and matched normal controls had similar socioeconomic status and dietary patterns.

\section{Clinical Measures}

Patient psychopathology was assessed on the day of the blood sampling using the Positive and Negative Symptom Scale (PANSS), which was measured by two psychiatrists who had simultaneously attended a training session in the use of the PANSS before the study began. After training, repeated assessment showed that the two raters maintained a correlation coefficient greater than 0.8 for the PANSS total score.

Mean scores on the PANSS scale reflected a moderate severity of the illness with a predominance of negative symptoms. Mean scores were as follows: positive subscale, $16 \pm 7$; negative subscale, $25 \pm 7$; general subscale, $32 \pm 9$, and total PANSS score, $73 \pm 18$. Clinical subtypes of schizophrenia were as follows: paranoid, $78(40 \%)$; disorganized, $35(18 \%)$; undifferentiated, $22(11 \%)$; and residual schizophrenia, $61(31 \%)$.

\section{BDNF Measurement}

Serum samples were collected between 0700 and 0900 hours following an overnight fast. The serum was separated, aliquoted, and stored at $-70^{\circ} \mathrm{C}$ before use. To minimize the assay variance, serum BDNF levels were measured in duplicate in all subjects on the same day by sandwich ELISA using a commercially available kit (BanDing Biomedical Inc., Chinese Academy of Sciences, Beijing, China). A full description of the assays has been given in our previous report (Tan et al, 2005a,b). All samples were assayed by a technician blind to the clinical situation. The identity of all subjects was indicated by a code number maintained by the investigator until all biochemical analyses were completed. Inter- and intra-assay variation coefficients were 7 and 5\%, respectively. 
Table I Mean $( \pm S D)$ change in BMI and mean serum BDNF levels after long-term treatment with antipsychotics in schizophrenia according to BDNF genotype

\begin{tabular}{|c|c|c|c|c|c|c|c|c|}
\hline & Val/Val & $n$ & Met/Val & $n$ & Met/Met & $n$ & $\mathbf{F}$ & $p$ \\
\hline \multicolumn{9}{|c|}{ Change in BMl (kg/m2) } \\
\hline All patients & $2.1 \pm 2.4$ & 52 & $2.6 \pm 2.8$ & 105 & $5.2 \pm 3.9$ & 39 & 4.84 & 0.009 \\
\hline Male & $2.3 \pm 2.6$ & 36 & $1.7 \pm 2.4$ & 63 & $5.8 \pm 4.1$ & 31 & 5.48 & 0.004 \\
\hline Female & $2.0 \pm 2.1$ & 16 & $3.7 \pm 3.1$ & 42 & $4.6 \pm 3.7$ & 8 & 2.01 & 0.13 \\
\hline Male & $7.1 \pm 2.4$ & 36 & $7.9 \pm 3.3$ & 63 & $8.3 \pm 2.8$ & 31 & 1.34 & 0.27 \\
\hline Female & $8.4 \pm 4.2$ & 16 & $5.9 \pm 3.1$ & 42 & $4.8 \pm 1.3$ & 8 & 4.57 & 0.014 \\
\hline
\end{tabular}

Abbreviation: BDNF, brain-derived neurotrophic factor.

Data are mean (SD). BMI indicates body mass index.

\section{BDNF Genotyping}

DNA was extracted using standard protocols. The genotypes of the BDNF Val66Met polymorphisms were identified as reported earlier (Neves-Pereira et al, 2002). Briefly, a 113-bp segment was amplified by PCR, using the following primers: $5^{\prime}$-GAGGCTTGACATCATTGGCT-3' and 5'-CGTGTACAAG TCTGCGTCCT- ${ }^{\prime}$.

The Val66Met polymorphism was differentiated by Eco721 restriction enzyme. The fragments were separated on a $3.5 \%$ agarose gel at $100 \mathrm{~V}$, and fragments were visualized with ethidium bromide. The uncut product size was $113 \mathrm{bp}$ (allele A), and allele $\mathrm{G}$ comprised the cut bands of 78 and $35 \mathrm{bp}$. Genotyping was duplicated and carried out blind to the clinical status.

\section{Data Analysis}

All data were analyzed using SPSS 10.1 program for Windows. Associations of the three genotypes (based on the variant allele) with weight gain, expressed as change in BMI, and with BDNF levels were assessed using one-way analysis of variance, and when significant, the effects of age, sex, duration of illness, and neuroleptic dose were added as covariates in an analysis of co-variance. The relationships of serum BDNF levels with BMI change and the clinical variables were tested with Pearson product moment correlations. All associations were adjusted for possible confounding factors such as sex and age. Significance level was set at 0.05 .

\section{RESULTS}

\section{Association of BDNF Genotype with Weight Gain}

The genotypic distributions of BDNF genes in patients and normal controls were consistent with Hardy-Weinberg equilibrium (all $p>0.05$ ). There was no difference in BDNF genotype distribution between the patient and normal control groups (schizophrenia: Met/Met 26.5\%, Val/Met 53.6\%, Val/Val 19.9\%; normal controls: Met/Met 22.6\%, $\mathrm{Val} /$ Met 53.1\%, $\mathrm{Val} / \mathrm{Val} 24.3 \% ; p>0.05$ ) and also there was no relationship of BDNF genotypes to BMI in the normal control group nor to baseline BMI in the patient group (both $p>0.05$ ).

Mean weight gain in patients since the onset of their illness was $8.6 \pm 8.4 \mathrm{~kg}$ and mean BMI gain was $3.2 \pm 3.1 \mathrm{~kg} / \mathrm{m}^{2}$. The BDNF Met/Met group had significantly higher BMI gain than the group with Val allele $(\mathrm{F}=4.84, \mathrm{df}=2,193$, $p=0.01)$. The $\mathrm{Val} / \mathrm{Val}$ and $\mathrm{Val} / \mathrm{Met}$ groups showed no difference in BMI gain between them (Table 1).

The sex-by-genotype interaction was significant $(\mathrm{F}=3.39$, $\mathrm{df}=2,190, p=0.03)$, and separate analyses showed an effect of genotype on BMI gain in male $\left(p=0.004 ; r^{2}=0.14\right)$, but not female patients (Table 1). Males with the Met/Met genotype had significantly higher mean BMI gain than males with the Val allele (both $p<0.01$ ). The difference in mean BMI gain between the genotypes remained significant when controlling for all relevant variables including initial BMI, current BMI, age, the type of antipsychotic medication, duration of exposure to antipsychotics, exposure to psychotropic medications, duration of illness, and drug dose (both $p<0.05$ ).

In addition, a 1-1-1 matching of three genotypes in male or female patients was carried out thereby minimally reducing the sample size to $28-28-28$ in male patients or $8-8-8$ in female patients in a secondary analysis. This minimized the effects of unequal cell sizes, but the primary results of an effect of genotype on BMI gain in male but not female patients were unchanged (date not shown).

\section{Association of BDNF Genotype with Serum BDNF Levels}

Serum BDNF levels were significantly lower in patients compared to normal controls $(7.0 \pm 3.1$ vs $9.4 \pm 4.4 \mathrm{ng} / \mathrm{ml}$, $p<0.001)$, but there were no significant differences among the allelic groups. While males showed no significant effect of genotype on serum BDNF levels, females bearing the Met/ Met genotype had significantly lower mean BDNF levels than females bearing the Val allele (both $p<0.05$ ) (Table 1). However, serum BDNF levels did not differ between the genotypes when controlling for the relevant variables including initial BMI, current BMI, age, the type of antipsychotic medication, duration of exposure to antipsychotics, exposure to psychotropic medications, duration of illness, and drug dose. 

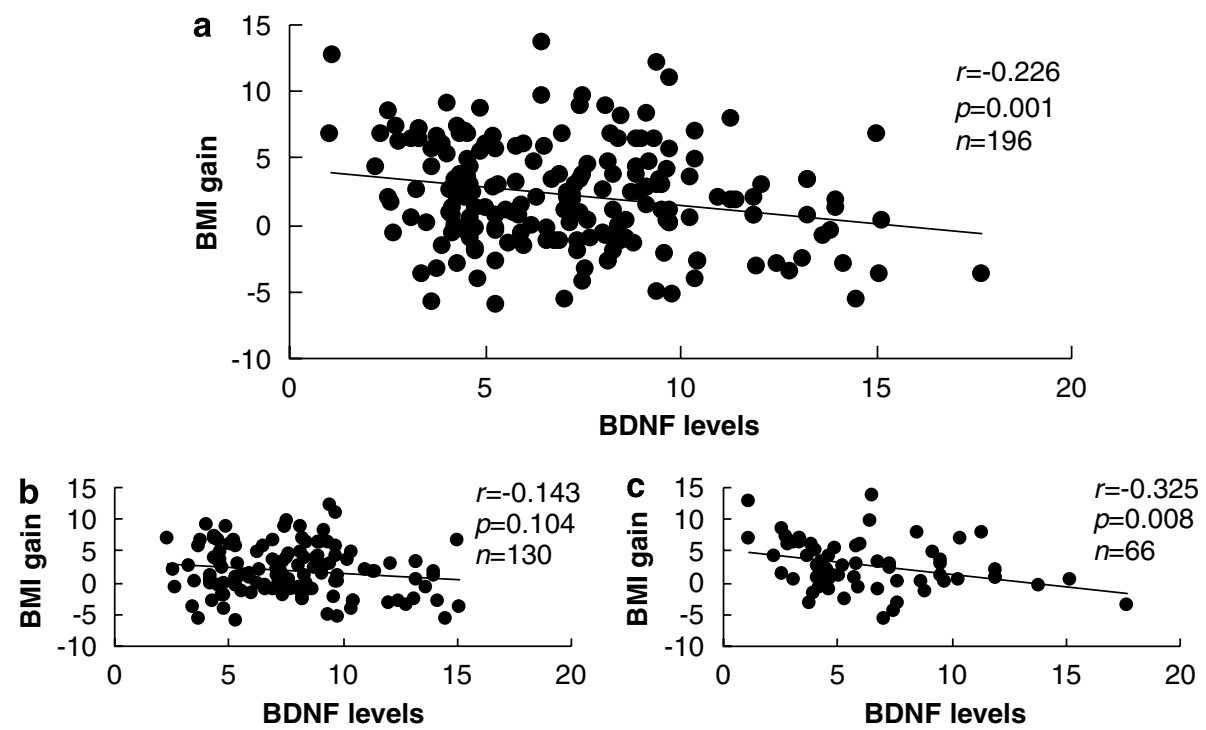

Figure I (a) Relationship between BDNF and BMI gain for all patients. (b) Relationship between BDNF and BMI gain for male patients. (c) Relationship between BDNF and BMI gain for female patients.

\section{Relation between Serum BDNF Levels and BMI Gain}

Serum BDNF levels and BMI gain were negatively correlated $(r=-0.23, n=196, p=0.001)$. This correlation was found in females $(r=-0.325, n=66, p=0.008)$, but not males (Figure 1). Controlling for initial BMI, current BMI, age, the type of antipsychotic medication, duration of exposure to antipsychotics, exposure to psychotropic medications, duration of illness, and drug dose did not eliminate these associations of BDNF and BMI gain.

\section{Relation between Serum BDNF Levels, Genotype, BMI Gain, and Clinical Variables}

Neither demographic, clinical variables (age of onset of the illness, duration of illness, and hospitalization) nor schizophrenia subtype had any association with BDNF genotype or serum levels. Also, antipsychotic dose (chlorpromazine equivalents), and the duration of taking neuroleptic medications and the anti-parkinsonian drugs did not show any association with BDNF genotype or serum levels. Furthermore, no significant correlation between BMI value or BMI gain and psychopathological symptoms on the PANSS total score or its subscores was observed. In addition, the different forms of antipsychotic medication did not have different effects on either BDNF or change in BMI (data not shown).

\section{DISCUSSION}

The major findings of the present study were as follows. (1) The Val66Met polymorphism in the BDNF promoter region correlated with weight gain after long-term antipsychotic treatment in chronic schizophrenic patients and (2) BDNF serum levels were associated with antipsychotic-induced weight gain in female patients with chronic schizophrenia. To our knowledge, this is the first study to examine the relationship between BDNF and antipsychotic-induced weight gain. Our findings suggest that individual differences in genetic alleles associated with BDNF may correlate with weight gain induced by antipsychotic drugs in schizophrenia.

Our results support the Val66Met polymorphism of the BDNF gene as a candidate marker for risk of neurolepticinduced weight gain among schizophrenic patients (Basile et al, 2001; Reynolds et al, 2002). The association was gender-specific and explained approximately $14 \%$ of the weight gain in the male patients. It also was consistent with expected gender differences in the genetic contribution to obesity (Hellstrom et al, 1999). The significant weight gain in the patients with the BDNF-Met 66 variant is also compatible with two recent studies showing that the Met66 BDNF variant is associated with eating disorders including anorexia nervosa and bulimia nervosa (Ribases et al, 2003, 2004). However, the mechanisms relating the BDNF Met-66 variant and weight gain are still unknown, and the association may reflect linkage disequilibrium with another functional polymorphism in the structural or regulatory region of the BDNF gene. Furthermore, other gene polymorphisms, such as those associated with the dopamine D3, 5-HT2C, and 5-HT2A receptors, may contribute additional pharmacodynamic effects, while genetic variation in drug metabolism enzymes such as the CYP family is probably responsible for pharmacokinetic effects (Reynolds et al, 2005).

Although mechanisms underlying the association of BDNF and weight gain are not clearly understood, many components for a reasonable mechanism have been found. Impairments in BDNF synthesis or production in animals are associated with increased food intake, reduced energy expenditure, and weight gain (Kernie et al, 2000; Lyons et al, 1999; Nakagawa et al, 2000; Nonomura et al, 2001; Ono et al, 1997; Rios et al, 2001; Tsuchida et al, 2001). In contrast, central infusions of BDNF in rats lead to severe, dose-dependent appetite suppression, weight loss, and increase in serotonin (Pelleymounter et al, 1995). 
Neuroanatomically, the ventromedial hypothalamus neurons that express BDNF importantly influence the regulation of energy balance through melanocortin-4 receptors (MC4R) (Xu et al, 2003). Expression of BDNF in ventromedial hypothalamus neurons is regulated by nutritional state and MC4R signaling. BDNF infusion into the brain suppresses the hyperphagia and excessive weight gain characteristic of heterozygous MC4R-deficient mice, a wellknown model for human obesity (Hinney et al, 2003). Taken together, these studies implicate BDNF in energy expenditure, energy balance, and proper weight maintenance (Kernie et al, 2000;Lyons et al, 1999). BDNF may directly reduce food consumption and body weight (Ribases et al, 2004), in addition to acting indirectly through serotonin (Kernie et al, 2000; Lyons et al, 1999; Ono et al, 1997; Rios et al, 2001). Thus, the lower serum BDNF levels in female schizophrenic patients who have antipsychoticinduced weight gain provides a plausible mechanism, but the lack of a low BDNF and weight gain association in males, in spite of a genetic association with weight gain in males, casts doubt on the low BDNF levels as a mediator or mechanism for this genetic polymorphism to directly influence risk for weight gain.

However, it is noteworthy whether serum levels of BDNF correlated with brain BDNF levels or activity. Recently, Karege et al (2002) reported that brain and serum BDNF levels underwent similar changes during maturation and aging process in rats. They also found a positive correlation between serum and cortical BDNF levels, suggesting that blood BDNF levels may reflect BDNF in the brain. However, the transport of BDNF via the blood-brain barrier is an important question as serum measurements were made in the study. Although there is an article available showing that BDNF from the blood stream could cross the blood-brain barrier under experimental conditions using mice model (Pan et al, 1998), there is no proof for such bidirectional transport of BDNF in humans. Therefore, whether a decrease in peripheral BDNF levels might possibly influence the cascade relevant in the pathophysiology of schizophrenia also in the central nervous system remains to be elucidated in further investigations.

The present study has several limitations. One limitation is that this sample of chronic in-patients had more severe psychopathology, and a longer duration of illness, treatment, and hospitalization than typical schizophrenic patients. Second, all patients had been on their current antipsychotic treatment for at least 2 years with a mean treatment duration of 6 years, but they also had been treated with different antipsychotic drugs over a 19-year course of illness. The weight gain attributed to current antipsychotic treatment may have been affected by previous antipsychotics. Third, relatively few of the patients were female, and the critical correlations between BMI gain and BDNF levels were restricted to the female patients. Hence, our results need replication in studies with more females.

\section{ACKNOWLEDGEMENTS}

This study was funded by the Stanley Medical Institute Foundation (03T-459, 05T-726) (XYZ), the National Basic Research Program of China (973 Program; 2007BC512307)
(DFZ), and the Department of Veterans Affairs, VISN 16, Mental Illness Research, Education and Clinical Center (MIRECC) and National Institute on Drug Abuse K05-DA0454 and P50-DA18827 (TRK).

\section{DISCLOSURE/CONFLICT OF INTEREST}

The authors have no conflicts to disclose.

\section{REFERENCES}

Allison DB, Casey DE (2001). Antipsychotic-induced weight gain: a review of the literature. J Clin Psychiatry 62(Suppl 7): 22-31.

Allison DB, Mentore JL, Heo M, Chandler LP, Cappelleri JC, Infante MC et al (1999). Antipsychotic-induced weight gain: a comprehensive research synthesis. Am J Psychiatry 156: 1686-1696.

American Psychiatric Association (1994). Diagnostic and Statistical Manual of Mental Disorders, 4th edn, American Psychiatric Association, Washington, D.C.

Angelucci F, Mathe AA, Aloe L (2000). Brain-derived neurotrophic factor and tyrosine kinase receptor $\operatorname{TrkB}$ in rat brain are significantly altered after haloperidol and risperidone administration. J Neurosci Res 60: 783-794.

Baptista T (1999). Body weight gain induced by antipsychotic drugs: mechanisms and management. Acta Psychiatr Scand 100: 3-16.

Basile VS, Masellis M, McIntyre RS, Meltzer HY, Lieberman JA, Kennedy JL (2001). Genetic dissection of atypical antipsychoticinduced weight gain: novel preliminary data on the pharmacogenetic puzzle. J Clin Psychiatry 62(Suppl 23): 45-66.

Blackburn GL (2000). Weight gain and antipsychotic medication. J Clin Psychiatry 61(Suppl 8): 36-41.

Correll CU, Malhotra AK (2004). Pharmacogenetics of antipsychotic-induced weight gain. Psychopharmacology 174: 477-489.

Egan MF, Kojima M, Callicott JH, Goldberg TE, Kolachana BS, Bertolino A et al (2003). The BDNF val66met polymorphism affects activity-dependent secretion of BDNF and human memory and hippocampal function. Cell 112: 257-269.

Hellstrom L, Large V, Reynisdottir S, Wahrenberg H, Arner P (1999). The different effects of a Gln27Glu beta 2-adrenoceptor gene polymorphism on obesity in males and in females. J Intern Med 245: 253-259.

Hinney A, Hohmann S, Geller F, Vogel C, Hess C, Wermter AK et al (2003). Melanocortin-4 receptor gene: case-control study and transmission disequilibrium test confirm that functionally relevant mutations are compatible with a major gene effect for extreme obesity. J Clin Endocrinol Metab 88: 4258-4267.

Karege F, Schwald M, Cisse M (2002). Postnatal developmental profile of brain-derived neurotrophic factor in rat brain and platelets, Neurosci. Lett 328: 261-264.

Kernie SG, Liebl DJ, Parada LF (2000). BDNF regulates eating behavior and locomotor activity in mice. EMBO J 19: 1290-1300.

Lipska BK, Khaing ZZ, Weickert CS, Weinberger DR (2001). BDNF mRNA expression in rat hippocampus and prefrontal cortex: effects of neonatal ventral hippocampal damage and antipsychotic drugs. Eur J Neurosci 14: 135-144.

Lyons WE, Mamounas LA, Ricaurte GA, Coppola V, Reid SW, Bora SH (1999). Brain-derived neurotrophic factor-deficient mice develop aggressiveness and hyperphagia in conjunction with brain serotonergic abnormalities. Proc Natl Acad Sci USA 96: 15239-15244.

Nakagawa T, Tsuchida A, Itakura Y, Nonomura T, Ono M, Hirota F et al (2000). Brain-derived neurotrophic factor regulates glucose metabolism by modulating energy balance in diabetic mice. Diabetes 49: 436-444. 
Nasrallah H (2003). A review of the effect of atypical antipsychotics on weight. Psychoneuroendocrinology 28(Suppl 1): 83-96.

Neves-Pereira M, Mundo E, Muglia P, King N, Macciardi F, Kennedy JL (2002). The brain derived neurotrophic factor gene confers susceptibility to bipolar disorder: evidence from a family-based association study. Am J Hum Genet 71: 651-655.

Nonomura T, Tsuchida A, Ono-Kishino M, Nakagawa T, Taiji M, Noguchi H (2001). Brain-derived neurotrophic factor regulates energy expenditure through the central nervous system in obese diabetic mice. Int J Exp Diabetes Res 2: 201-209.

Ono M, Ichihara J, Nonomura T, Itakura Y, Taiji M, Nakayama C et al (1997). Brain derived neurotrophic factor reduces blood glucose level in obese diabetic mice but not in normal mice. Biochem Biophys Res Commun 238: 633-637.

Pan W, Banks WA, Fasold MB, Bluth J, Kastin AJ (1998). Transport of brain-derived neurotrophic factor across the blood-brain barrier. Neuropharmacology 37: 1553-1561.

Pelleymounter MA, Cullen MJ, Baker MB, Hecht R, Winters D, Boone $\mathrm{T}$ et al (1995). Effects of the obese gene product on body weight regulation in ob/ob mice. Science 269: 540-543.

Reynolds GP, Templeman LA, Zhang ZJ (2005). The role of 5-HT2C receptor polymorphisms in the pharmacogenetics of antiphsychotic drug treatment. Prog Neuropsychopharmacol Biol Psychiatry 29: 1021-1028.

Reynolds GP, Zhang ZJ, Zhang XB (2002). Association of antipsychotic drug-induced weight gain with a 5-HT2C receptor gene polymorphism. Lancet 359: 2086-2087.

Ribases M, Gratacos M, Armengol L, de Cid R, Badia A, Jimenez L et al (2003). Met66 in the brain-derived neurotrophic factor
(BDNF) precursor is associated with anorexia nervosa restrictive type. Mol Psychiatry 8: 745-751.

Ribases M, Gratacos M, Fernandez-Aranda F, Bellodi L, Boni C, Anderluh $\mathrm{M}$ et al (2004). Association of BDNF with anorexia, bulimia and age of onset of weight loss in six European populations. Hum Mol Genet 13: 1205-1212.

Rios M, Fan G, Fekete C, Kelly J, Bates B, Kuehn R et al (2001). Conditional deletion of brain-derived neurotrophic factor in the postnatal brain leads to obesity and hyperactivity. Mol Endocrinol 15: 1748-1757.

Tan YL, Zhou DF, Cao LY, Zou YZ, Zhang XY (2005a). Decreased BDNF in serum of patients with chronic schizophrenia on longterm treatment with antipsychotics. Neurosci Lett 382: 27-32.

Tan YL, Zhou DF, Zhang XY (2005b). Decreased plasma brain derived neurotrophic factor levels in schizophrenic patients with tardive dyskinesia: association with dyskinetic movements. Schizophr Res 74: 263-270.

Taylor DM, McAskill R (2000). Atypical antipsychotics and weight gain - a systematic review. Acta Psychiatr Scand 101: 416-432.

Tsuchida A, Nonomura T, Ono-Kishino M, Nakagawa T, Taiji M, Noguchi H (2001). Acute effects of brain-derived neurotrophic factor on energy expenditure in obese diabetic mice. Int $J$ Obes Relat Metab Disord 25: 1286-1293.

Werneke U, Taylor D, Sanders TA (2002). Options for pharmacological management of obesity in patients treated with atypical antipsychotics. Int Clin Psychopharmacol 17: 145-160.

$\mathrm{Xu} \mathrm{B}$, Goulding EH, Zang K, Cepoi D, Cone RD, Jones KR et al (2003). Brain-derived neurotrophic factor regulates energy balance downstream of melanocortin-4 receptor. Nat Neurosci 6: $736-742$. 